\title{
EDITORIAL
}

\section{The fate of respiratory physiology}

\author{
P.J. Barnes*
}

Respiratory physiology is under threat. What was once regarded as the science underpinning respiratory medicine is now being eroded by a move towards cell and molecular biology that threatens its very existence. This trend is not confined to the respiratory field, but is also occurring in most other specialities. Some academic departments of respiratory physiology and research groups in physiology are already under threat of closure, or are being drastically reduced to make way for more molecular biologists (deoxyribonucleic acid (DNA) biochemists). As I write this editorial, one of the most prestigious and productive respiratory physiology departments in the world, the Department of Physiology in the University of Leiden, led by Professor Philip Quanjer, is about to be dismantled. The time has come to halt the erosion of physiology, before we lose a generation of trained individuals. As molecular biology advances, never has the need for integrative physiology been greater. Without innovative respiratory physiology, some of the most interesting questions posed by molecular and cell biology cannot be answered, and the enormous potential of the advances in cell and molecular biology cannot be fully realized.

Molecular biology has grown enormously over the last $20 \mathrm{yrs}$ and has provided techniques that can answer questions previously unimaginable. The attraction of molecular biology is that it will enable us to understand all human disease at a molecular level and, thus, lead to more specific treatments in the future. This is the rationale for the enormous expenditure on the Human Genome Project. The identification of particular genes has made it possible to study the genetic basis of disease, and to elucidate the detailed structures of important proteins, such as enzymes, receptors and signalling proteins. Many of the advances in molecular biology have relied upon cultured cell lines, which make it possible to study expression of genes and the factors that regulate their expression in great detail. However, there is increasing recognition that this approach may have limited application, because many cell lines do not function in the same way as cells in the living organism, and they are not subject to the same regulatory control mechanisms. This means that control mechanisms in the intact animal may be quite different to those identified in cell lines. Another problem that is becoming ever more apparent is that studying the regulation of gene expression by measuring the steady-state messenger ribonucleic acid (mRNA) content of a cell (by Northern blotting or solution hybridization), or by directly measuring the rate of gene transcription (by nuclear run-on assays), does not necessarily predict what will happen to the production of the protein product of the gene or its function, since several changes may occur in the mRNA level before the protein is translated, and changes may also occur after translation of the *Dept of Thoracic Medicine, National Heart and Lung Institute, London SW3 6LY, UK. protein in the cytoplasm. This is now leading to the view that molecular biology in isolation is inadequate, and that to conduct molecular biology in isolation, without concomitant measurements of protein expression and function, may be misleading.

Molecular and cell biology (the "new biology") are seductive because they offer so much. Unfortunately, this has persuaded national funding bodies to finance this research in favour of more traditional physiological and pharmacological approaches, from a diminishing pot of money. Physiology and pharmacology have become unfashionable. This short-sighted and unwise approach has led to the atrophy of physiology and pharmacology departments in universities. This attitude has also filtered down to students, who avidly study the new biology but are not taught the basic principles of physiology and pharmacology. This will lead to a generation uneducated in these important sciences, at a time when there will be the most need for these skills. Molecular biology does have enormous potential for increasing our understanding of all respiratory diseases (see [1]), but it will not be possible to take advantage of these approaches without integrating molecular biology into whole organ physiology, particularly in humans. There will be an increasing need to develop and apply physiology in order to exploit the fruits of molecular biology.

Molecular techniques have commonly been applied to isolated or cultured cells because of the nature of the technology currently available. While this may give detailed information about a single cell (or transformed cell line), this approach misses the complexities of cell-to-cell interactions, which are the essence of complex organisms. The philosophy of integrative physiology is that the whole is more than the sum of its individual parts. This means that, in the future, the communication between cells and the control mechanisms regulating integrated responses must be taken into account, particularly when trying to elucidate dysfunction in disease. This is where physiology has much to teach molecular biology and in the future it will be necessary to apply molecular and cell biology techniques in the intact organism, where it will be important to make careful measurements of function. With understanding of the detailed function of individual parts we may still not understand how the intact human functions. What is needed for the future is the integration of molecular and cell biology with the approaches adopted in integrative physiology.

Molecular biology will provide new tools that can be used to explore the pathophysiology of respiratory diseases. These will include complementary deoxyribonucleic acid (cDNA) probes to examine the expression of particular genes, but this information must be linked to functional information. At the end of the day, it is the functional information that must take precedence; an alteration in expression of a particular gene that is without functional consequence may not be clinically relevant (and vice versa). 
Molecular biology will also make it possible to apply gene replacement therapy. The respiratory tract may be particularly suitable for testing gene therapy, as the replacement genes may be applied to the airway surface. Indeed, cystic fibrosis is likely to be one of the first human diseases where gene therapy is tested. This provides a good example of how important respiratory physiology will be in the future, since the functional efficacy of replacement of the cystic fibrosis transmembrane conductance regulator (CFTR) gene, that is defective in cystic fibrosis, either by an adenovirus or liposome vector, will need to be measured. This can be done by monitoring epithelial potential difference, a method dependent on advances in respiratory physiology. The follow-up of cystic fibrosis treatments will need to be made by careful physiological measurements of airway function and airway secretions. Similar considerations will apply equally to other diseases where gene therapy is contemplated, such as emphysema due to $\alpha_{1}$-proteinase inhibitor deficiency [1].

Another example of the importance of the link between molecular biology and respiratory physiology is provided by recent work on nitric oxide (NO). NO is produced by the enzyme NO synthase, of which several isoforms are now recognized and have been cloned. Of particular interest to airway disease is the inducible form of NO synthase (iNOS), which is induced in several cell types by exposure to proinflammatory cytokines. This enzyme can be induced in epithelial cells [2], and is expressed in epithelial cells of asthmatic but not normal airways [3], presumably in response to local cytokine production. NO is present in the exhaled air of normal individuals [4], and there is an increased amount in the exhaled air of patients with asthma [5]. Measurement of exhaled NO may, therefore, provide an indication of iNOS gene expression in the airways and, thus, of cytokine-mediated inflammation in the respiratory tract. Interpretation of the measurement of exhaled NO is now an important question for respiratory physiology, as this may provide a window on airways inflammation.

Animal research has allowed us to explore normal physiology and models of lung disease in great detail. Molecular and cell biology techniques can be applied to animals in vivo, in order to integrate these approaches. Unfortunately, this is rarely done, as molecular biologists and animal physiologist may not communicate. Animal respiratory physiology offers sophisticated techniques that provide an opportunity to study the in vivo implications of discoveries made in cell lines and in in vitro systems. The ability to develop transgenic animals that either over-express a particular gene or have the gene deleted ("knock-out" animals) has been a major advance, and is revealing much about the function of specific genes in vivo (usually in mice). In order to study the function of genes in the lung in vivo, respiratory physiological techniques are essential. Of course, for many diseases, such as asthma, emphysema and fibrosing lung diseases, there are no satisfactory animal models. For this reason, it is important to develop human respiratory physiology.

Another area that may become increasingly important is the potential provided by antisense and antigene drugs [6]. Antisense oligonucleotides are short oligonucleotides (18-20 nucleotide bases is optimal) that are complementary to a part of the sequence of mRNA and, thus, inhibit the synthesis of a particular protein. Antigene oligonucleotides are complementary to the gene sequence and bind to the double-stranded DNA sequence, thus preventing gene transcription. These oligonucleotides are, thus, able to prevent the synthesis of proteins in a highly specific way, and are now being developed as very specific blocking drugs. The major disadvantage of oligonucleotides is poor cell penetration and degradation by nucleases, but modification of the nucleotides overcomes this problem, and may lead to the development of stable drugs that are effective in animals in vivo. The potential for such drugs is enormous, both in studying disease mechanisms and in providing new and more specific therapies. This approach offers obvious advantages over the "knock-out" gene models in mice, where a selected gene is disrupted in mouse embryonic cells by replacement with DNA lacking the gene of interest. These techniques are difficult technically and are inconceivable in humans. Delivery of antisense drugs to the respiratory tract may be an early application of this new approach and, as in the other examples cited above, the development of suitable respiratory physiology techniques to monitor their efficacy will be of vital importance.

The time has come to prevent the erosion of respiratory physiology before it is too late. It needs to be recognized that respiratory physiology and the development of new physiological approaches will be of critical importance in harnessing the new biology to respiratory medicine. Respiratory diseases are the commonest cause of morbidity worldwide, and asthma is the commonest chronic disease in industrialized countries. Respiratory diseases impose an enormous financial burden universally, and deserve to be taken seriously. It would be quite inappropriate to start dismantling the very props that support this speciality. The respiratory tract provides an ideal test-bed for some of the developments from molecular biology, and it is no coincidence that cystic fibrosis is amongst the first diseases in which gene therapy will be tested. Respiratory physiology has provided some remarkably sensitive techniques to monitor pulmonary function in health and disease. There needs to be a recognition by funding bodies and by administrators that the integration of the new biology with physiology is essential, if we are to interpret and to take advantage of the advances in basic science. Physiologists must stand up and be counted!

\section{References}

1. Barnes PJ, Stockley RA. Molecular biology of lung disease. Oxford, Blackwell 1994.

2. Robbins RA, Springall DR, Warren JB, et al. Inducible nitric oxide synthase is increased in murine lung epithelial cells by cytokine stimulation. Biochem Biophys Res Commun 1994; 198: 1027-1033.

3. Hamid Q, Springall DR, Riveros-Moreno V, et al. Induction of nitric oxide synthase in asthma. Lancet 1993; 342: $1510-1513$.

4. Gustafsson LE, Leone AM, Persson M-G, Wiklund NP, Moncada S. Endogenous nitric oxide is present in the exhaled air of rabbits, guinea-pigs and humans. Biochem Biophys Res Commun 1991; 181: 852-857.

5. Kharitonov SA, Yates D, Robbins RA, Logan-Sinclair $\mathrm{R}$, Shinebournes E, Barnes PJ. Increased nitric oxide in exhaled air of asthmatic patients. Lancet 1994; 34 : 133-135.

6. Wahlestedt C. Antisense oligonucleotide strategies in neuropharmacology. Trend Pharmacol Sci 1994; 15: 42-45. 